\title{
BAIRE PARADOXICAL DECOMPOSITIONS NEED AT LEAST SIX PIECES
}

\author{
FRIEDRICH WEHRUNG
}

(Communicated by Franklin D. Tall)

\begin{abstract}
We show that in certain cases paradoxical decompositions of compact metric spaces using sets (or even [0,1]-valued functions) with the property of Baire modulo meager sets need more pieces than paradoxical decompositions with unrestricted pieces. In particular, any Baire paradoxical decomposition of the sphere $S^{2}$ using isometries needs at least six pieces.
\end{abstract}

Let $G$ be a group of isometries of a compact metric space $(X, d)$. Denote by $\mathscr{B}(X)$ the additive semigroup of real-valued bounded positive functions on $X$ that have the Baire property (see [2] for this and related terminology, e.g., first category, Baire category theorem, etc.). If $\varphi$ and $\psi$ are two elements of $\mathscr{B}(X), \varphi \geq \psi$ (B-a.e.) will mean that the set of all $x$ such that $\varphi(x) \geq \psi(x)$ is of first category and similarly for $\varphi=\psi$ (B-a.e.). There is a canonical action of $G$ on $\mathscr{B}(X)$ defined by $(g \varphi)(x)=\varphi\left(g^{-1} x\right)$. Two elements $\varphi$ and $\psi$ of $\mathscr{B}(X)$ are said to be (continuously) G-equidecomposable (see [4]) when there are $n$ in $\mathbb{N}, \varphi_{1}, \ldots, \varphi_{n}$ in $\mathscr{B}(X)$, and $g_{1}, \ldots, g_{n}$ in $G$ such that

$$
\varphi=\sum_{i=1}^{n} \varphi_{i} \quad \text { (B-a.e.) } \quad \text { and } \quad \psi=\sum_{i=1}^{n} g_{i} \varphi_{i} \quad \text { (B-a.e.). }
$$

When the $\varphi_{i}$ are restricted to be $\{0,1\}$-valued functions, this is the usual notion of Baire equidecomposability, which we shall call discrete, as opposed to continuous (see [4]). In general, continuous equidecomposability is weaker than discrete equidecomposability. We will say that $\varphi$ is Baire paradoxical (with respect to the group $G$ ) when $\varphi$ and $2 \varphi$ are equidecomposable in $\mathscr{B}(X)$; we will emphasize "continuous" or "discrete" if the context does not make it clear. If $Y \subseteq X$, we will identify $Y$ with its characteristic function $\mathbf{1}_{Y}$.

Lemma. Let $\varphi$ in $\mathscr{B}(X)$ and $g$ in $G$ be such that $g \varphi \geq \varphi$ (B-a.e.). Then $g \varphi=\varphi \quad(B$-a.e.).

Proof. There is a comeager subset $Y$ of $X$ such that the restriction $\left.\varphi\right|_{Y}$ is continuous (see [2]); furthermore, one can suppose without loss of generality that $g Y=Y$ and that $(\forall x \in Y)\left(\varphi\left(g^{-1} x\right) \geq \varphi(x)\right)$. We prove that for all $x$

Received by the editors September 16, 1992 and, in revised form, January 4, 1993.

1991 Mathematics Subject Classification. Primary 54E52; Secondary 54E45.

Key words and phrases. Paradoxical decomposition, Baire category, Baire property. 
in $Y$, we have in fact $\varphi\left(g^{-1} x\right)=\varphi(x)$. For, let $\left(g^{-n_{k}} x\right)_{k \in \mathbb{N}}$ be a convergent subsequence of $\left(g^{-n} x\right)_{n \in \mathbb{N}}$ and put $m_{k}=n_{k+1}-n_{k}$ so that $m_{k}>0$. Since $g$ is an isometry, we have $x=\lim _{k \rightarrow \infty} g^{-m_{k}} x$; since $\left.\varphi\right|_{Y}$ is continuous, it follows that $\varphi(x)=\lim _{k \rightarrow \infty} \varphi\left(g^{-m_{k}} x\right)$. But for all $k$ we have $\varphi\left(g^{-m_{k}} x\right) \geq$ $\varphi\left(g^{-1} x\right) \geq \varphi(x)$, whence $\varphi\left(g^{-1} x\right)=\varphi(x)$.

As a possible application, we get, for example, the following

Corollary. Let $m, n \in \mathbb{N}, g_{i} \in G$, and $\varphi_{i} \in \mathscr{B}(X) \quad(1 \leq i \leq m+n)$ such that

$$
1_{X}=\sum_{i=1}^{m+n} \varphi_{i}=\sum_{i=1}^{m} g_{i} \varphi_{i}=\sum_{i=m+1}^{m+n} g_{i} \varphi_{i} \quad \text { (B-a.e.). }
$$

Then $m \geq 3$ and $n \geq 3$.

Thus, if $X$ is Baire paradoxical, then any Baire-paradoxical decomposition of $X$ uses at least six pieces; of course, this works as well for continuous as for discrete equidecomposability. Furthermore, this has been recently shown to be possible in a very general context by Dougherty and Foreman [1]; in particular, $S^{2}$ is (discretely) Baire paradoxical using six pieces with respect to the group $\mathrm{SO}_{3}$ of rotations of $\mathbb{R}^{3}$ leaving the origin fixed. Thus, six is the optimal number of pieces required to realize a Baire-paradoxical decomposition of $S^{2}$.

Proof. Suppose, for example, that $m=2$. Furthermore, without loss of generality, $g_{1}=1$. Thus $g_{2} \varphi_{2}=1_{X}-\varphi_{1}=\sum_{i=2}^{m+n} \varphi_{i} \geq \varphi_{2}$ (B-a.e.), whence $g_{2} \varphi_{2}=\varphi_{2}$ (B-a.e.) by the lemma. Thus $\varphi_{i}=0$ (B-a.e.) for all $i \geq 3$, which contradicts the fact that $X$ is not of first category in itself.

Note that the same argument generalizes (with the same proof) to the case where $X$ is a metric space which is precompact (i.e., for each $r>0$ it can be covered by finitely many balls of radius $r$ )-so that convergent sequences are replaced by Cauchy sequences in the proof of the lemma-and which is not of first category in itself.

This result has to be put in contrast with the fact that $S^{2}$ is paradoxical using four, and not fewer than four, pieces (which do not necessarily satisfy the Baire property) (see [3]). Thus it is (much!) more difficult to realize paradoxes with pieces satisfying the property of Baire (even, as in this paper, modulo meager sets) than paradoxes with unrestricted pieces.

\section{REFERENCES}

1. R. Dougherty and M. Foreman, Banach-Tarski decompositions using pieces with the property of Baire, Proc. Nat. Acad. Sci. U.S.A. 89 (1992), 10726-10728.

2. J. Oxtoby, Measure and category, Springer-Verlag, New York, 1971.

3. S. Wagon, The Banach-Tarski paradox, Cambridge Univ. Press, Cambridge, New York, 1985.

4. F. Wehrung, Théorème de Hahn-Banach et paradoxes continus ou discrets, C. R. Acad. Sci. Paris Sér. I Math. 310 (1990), 303-306.

Département de Mathématiques, Université de Caen, 14032 Caen Cedex, France

E-mail address: gremlin@math.unicaen.fr 